\title{
Research Paper \\ Investigating the Meaning of Life and Psychological Well-being, in Youth, Adults, and Elderly (A Comparative Study of Three Age Groups)
}

\author{
*Soheila Orang ${ }^{1}$, Hadi Hashemi Razini ${ }^{2}$, Maryam Ramshini ${ }^{3}$
}

1. Department of Educational Psychology, Faculty of Psychology and Education, International Campus, Kharazmi Univarsity, Tehran, Iran.

2. Department of Psychology, Faculty of Psychology and Education, International Campus, Kharazmi Univarsity, Tehran, Iran.

3. Department of Psychology, Faculty of Literature, Humanities and Social Sciences, Science and Research Branch, Islamic Azad University, Tehran, Iran.

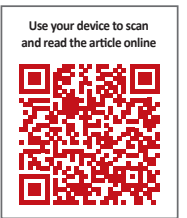

Citation: Orang S, Hashemi Razini H, Ramshini M. [Investigating the Meaning of Life and Psychological Well-being, in Youth, Adults, and Elderly (A Comparative Study of Three Age Groups) (Persian)]. Iranian Journal of Ageing. 2018; 13(2):182-197. https:// doi.org/10.32598/sija.13.2.182

doi : https://doi.org/10.32598/sija.13.2.182

Received: 03 Dec 2017

Accepted: 26 Apr 2018

Key words:

Elderly, Youth, Adults, The meaning of life, Psychological well-being

\section{A B STRACT}

Objectives The present study aimed to examine the meaning in life and well-being of young, middleaged, and older adults.

Methods \& Materials In this research, a total of 215 participants including 84 young (aged 17-25 years), 59 middle-aged (aged 26-46 years), and 72 older adults (aged 65-80 years) were selected from Tehran city via purposive sampling. The instruments used in this study consisted of the meaning of life questionnaire and Ryffs Scales of Psychological Well-being.

Results The results of Mancova analyses showed significant differences between the three age groups. The older people had the most ability in the meaning in life, self-acceptance, positive relation with others, personal growth and purpose in life.

Conclusion Research findings indicate that age affects the meaning in life and well- being. So, elderly can be known as a self-awareness, development and relaxation.

\section{Extended Abstract}

\section{Objectives}

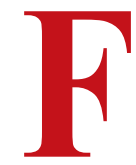

inding meaning in life and psychological well-being are considered as the most important factors in mental health and happiness. Given the negative attitudes and schemas regarding old age, it seems that young age and adulthood are preferred to the old age in terms of intelligence and finding meaning in life, mental health, the quality of life, and psychological well-being. Therefore, the present study has been conducted with the aim of comparing the meaning of life and psychological well-being of the elderly with those of young people and adults. Comparing these variables, this study tried to find out if old age limitations have led to reduced meaning of life and psychological well-being and whether young people and adults who are not bound by these restrictions have more meaning of life and psychological well-being. According to this, the main question of the present study is whether the age factor plays a role in

\section{* Corresponding Author:}

Soheila Orang, MSc.

Address: Department of Educational Psychology, Faculty of Psychology and Education, International Campus, Kharazmi Univarsity, Tehran, Iran. Tel: +98 (912) 6968611

E-mail: orang.soheila@gmail.com 
the meaning of life and psychological well-being of individuals in different age groups?

\section{Methods and Materials}

The present study is a causal-comparative study, and Multivariate Analysis of Variance (MANOVA) was used for data analysis while observing the assumptions. Statistical population consisted of all elderly people between the ages of 65 and 80 years, adults aged 26 to 46 years, and the youth between 17 and 25 years of age. In this study, 215 people were selected through non-random sampling, including 3 groups of youth, adults and elderly people. Data collection was carried out in the summer and autumn of 2016 in public places of Tehran. Inclusion criteria for the study were as follows: education level of diploma or above, the abovementioned age range, informed consent to answer the questions in the questionnaire, and the ability to understand questions. Exclusion criteria were refusal to continue participation and the inability to understand the questions. This study is a research type. Instruments used in this research were Reef's Psychological Well-being Scale and the Stareg's Meaning in Life Scale. In order to standardize the well-being scale in Iran, the coefficient of test-retest reliability for Reef's Psychological Well-being Scale was obtained to be $82 \%$. The reliability coefficients of its subscales of acceptance, the positive relationship with others, self-determination, objective life, personal growth, and environmental domination were $71 \%, 77 \%, 78 \%$, $70 \%, 77 \%$, and $78 \%$, respectively. The validity of this scale for life assessment was $86 \%$ for the sub-scale of the existence of meaning and $87 \%$ for the subscale of finding meaning in life. The reliability of sub-scales of existence of meaning and finding meaning in life were $70 \%$ and $73 \%$, respectively. Test-retest reliability of this scale in Iran was obtained to be $84 \%$ and $74 \%$ for subscales of existence of meaning and finding meaning in life, respectively, by Eshtehard (2009) with a two-

Table 1. Mean and standard deviation

\begin{tabular}{|c|c|c|c|}
\hline Components & Age & Mean & Standard Deviation \\
\hline & $17-25$ & 28.320 & 6.372 \\
\hline \multirow[t]{3}{*}{ Finding meaning } & $26-46$ & 27.849 & 4.959 \\
\hline & $65-80$ & 27.683 & 7.663 \\
\hline & $17-25$ & 23.615 & 3.661 \\
\hline \multirow[t]{3}{*}{ Existence of meaning } & $26-46$ & 23.150 & 3.575 \\
\hline & $65-80$ & 27.000 & 11.514 \\
\hline & $17-25$ & 11.551 & 1.904 \\
\hline \multirow[t]{3}{*}{ Self-acceptance } & $26-46$ & 12.132 & 1.605 \\
\hline & $65-80$ & 13.450 & 3.175 \\
\hline & $17-25$ & 8.294 & 3.338 \\
\hline \multirow[t]{3}{*}{ Positive relationship with others } & $26-46$ & 9.264 & 3.274 \\
\hline & $65-80$ & 12.300 & 3.646 \\
\hline & $17-25$ & 13.205 & 2.414 \\
\hline \multirow[t]{3}{*}{ Autonomy } & $26-46$ & 13.434 & 2.349 \\
\hline & $65-80$ & 12.850 & 2.735 \\
\hline & $17-25$ & 13.102 & 2.035 \\
\hline \multirow[t]{3}{*}{ Domination on environment } & $26-46$ & 13.905 & 1.86 \\
\hline & $65-80$ & 13.550 & 3.20 \\
\hline & $17-25$ & 9.897 & 2.515 \\
\hline \multirow[t]{3}{*}{ Purposeful life } & $26-46$ & 10.018 & 2.872 \\
\hline & $65-80$ & 12.266 & 3.085 \\
\hline & $17-25$ & 12.448 & 1.951 \\
\hline \multirow[t]{2}{*}{ Personal growth } & $26-46$ & 12.434 & 1.704 \\
\hline & $65-80$ & 14.466 & 7.729 \\
\hline
\end{tabular}


Table 2. Scheffe's follow-up test results

\begin{tabular}{|c|c|c|c|c|c|}
\hline Components & Age & Age & Mean Difference & Standard Error & Level of Significance \\
\hline \multirow{8}{*}{$\begin{array}{l}\text { Existence of mean- } \\
\text { ing }\end{array}$} & \multirow{3}{*}{$17-25$} & $26-46$ & 0.4644 & 1.266 & 0.935 \\
\hline & & & & & \\
\hline & & $65-80$ & -3.3846 & 1.221 & 0.023 \\
\hline & \multirow{2}{*}{$26-46$} & $17-25$ & -0.4644 & 1.266 & 0.935 \\
\hline & & $65-80$ & -3.8991 & 1.341 & 0.018 \\
\hline & \multirow{3}{*}{$65-80$} & $17-25$ & 3.3846 & 1.221 & 0.023 \\
\hline & & & & & \\
\hline & & $26-46$ & 3.8491 & 1.341 & 0.018 \\
\hline \multirow{8}{*}{ Self-acceptance } & \multirow{3}{*}{$17-25$} & $26-46$ & -0.5880 & 0.4122 & 0.373 \\
\hline & & & & & \\
\hline & & $65-80$ & -1.8987 & 0.3976 & 0.001 \\
\hline & \multirow{3}{*}{$26-46$} & $17-25$ & 0.5808 & 0.4122 & 0.373 \\
\hline & & & & & \\
\hline & & $65-80$ & -1.3179 & 0.4365 & 0.012 \\
\hline & \multirow[b]{2}{*}{$65-80$} & $17-25$ & 1.8987 & 0.3976 & 0.001 \\
\hline & & $26-46$ & 1.3179 & 0.4365 & 0.012 \\
\hline \multirow{8}{*}{$\begin{array}{l}\text { Positive relationship } \\
\text { with others }\end{array}$} & \multirow{3}{*}{$17-25$} & $26-46$ & -0.9693 & 0.6077 & 0.283 \\
\hline & & & & & \\
\hline & & $65-80$ & -4.0051 & 0.5862 & 0.001 \\
\hline & \multirow{2}{*}{$26-46$} & $17-25$ & 0.9693 & 0.6077 & 0.283 \\
\hline & & $65-80$ & -3.0358 & 0.6435 & 0.001 \\
\hline & \multirow{3}{*}{$65-80$} & $17-25$ & 4.0051 & 0.5862 & 0.001 \\
\hline & & & & & \\
\hline & & $26-46$ & 3.0358 & 0.6435 & 0.001 \\
\hline \multirow{8}{*}{ Purposeful life } & \multirow{2}{*}{$17-25$} & $26-46$ & -0.1214 & 0.49911 & 0.971 \\
\hline & & $65-80$ & -2.3692 & 0.48146 & 0.001 \\
\hline & \multirow{3}{*}{$26-46$} & $17-25$ & 0.1214 & 0.49911 & 0.971 \\
\hline & & & & & \\
\hline & & $65-80$ & -2.2478 & 0.52853 & 0.001 \\
\hline & \multirow{3}{*}{$65-80$} & $17-25$ & 2.3692 & 0.48146 & 0.001 \\
\hline & & & & & \\
\hline & & $26-46$ & 2.2478 & 0.52853 & 0.001 \\
\hline \multirow{9}{*}{ Personal growth } & \multirow{3}{*}{$17-25$} & $26-46$ & 0.0148 & 0.87179 & 0.0001 \\
\hline & & & & & \\
\hline & & $65-80$ & -2.0179 & 0.7890 & 0.040 \\
\hline & \multirow{3}{*}{$26-46$} & $17-25$ & -0.0148 & 0.8179 & 0.0001 \\
\hline & & & & & \\
\hline & & $65-80$ & -2.0327 & 0.86617 & 0.066 \\
\hline & \multirow{3}{*}{$65-80$} & $17-25$ & 2.0179 & 0.7890 & 0.040 \\
\hline & & & & & \\
\hline & & $26-46$ & 2.0327 & 0.86617 & 0.066 \\
\hline
\end{tabular}


weeks interval. Cronbach's alpha for finding meaning in life sub-scale was $75 \%$ and for the sub-scale of having meaning was $78 \%$. The scale seems to have a good internal consistency. Collected data were analyzed using SPSS version 18 and using ANOVA.

\section{Results}

The results obtained showed that the dependent variables have normal distribution. One of the presuppositions of the MANOVA test is Leven's test. The Leven's test showed that $\mathrm{F}$ error level for all components in the Leven's test was higher than 0.01 . Therefore, there was no significant difference between the groups in terms of the error variance of the components of finding meaning in life, existence of meaning, self-acceptance, positive relationships, autonomy, environmental domination, purposeful life, and personal growth. Results obtained from multivariate analysis of variance based on the scale of the Wilks' Lambda $(\mathrm{P}=0.01, \mathrm{~F}<6.84)$ showed that there was a statistically significant difference between the linear mean of attempts for meaning, existence of meaning, self-acceptance, positive relationships, autonomy, environmental domination, purposeful life, and individual growth of the three age groups. Given the values of F, there was a statistically significant difference between the means of existence of meaning, self-acceptance, positive relationships, autonomy, environmental domination, purposeful life, and individual growth of the three age groups. But there was no significant difference between age groups in terms of the mean scores related to finding meaning in life, autonomy, and environmental domination.

The findings of the present study showed that there was a significant difference between the age groups of $25-46,17-25$ and $65-80$ years in terms of the component of the existence of meaning. The findings indicate that the mean score of the existence of meaning in age group of 65-80 years was more than the two other age groups. There was a significant difference between groups of $17-25,25-46$ with $65-80$ in terms of self-acceptance. The mean score of the age group of 65-80 years for the component of self-acceptance was more than two other age groups. There was a significant difference between the age groups of 17-25 and 25-46 years compared to the age group of 65-80 years for the component of positive relationships with others. The mean score of the age group of 65-80 years for the positive relationship component was more than that of the two other age groups. There was a significant difference between the age groups of 17-25 years and 25-46 years with $65-80$ years in terms of the component of purposeful life. The mean score of purposeful life for the age group of 65-
80 years was more than two other age groups. There was a significant difference between the age groups of 17-25 years with the age group of 25-46 years in terms of the individual growth component. Mean score of age group of 65-80 years for the individual growth component was more than the age group of 17 to 25 years. Based on the findings obtained, the elderly people have a higher ability in terms of the existence of meaning, self-acceptance, positive relationship with others, individual growth and purposeful life (Tables 1 and 2).

\section{Conclusion}

From the present study, it can be concluded that the attitude towards life continued growth in different directions and dimensions. Based on the findings of this study, which indicates a significant difference in individual growth, purposeful life, positive relationship, self-acceptance, existence of meaning, and finding meaning in life for old age period, age can be an important factor in regulating emotions and reducing negative emotions. Therefore, it seems that the age factor increases the meaning of life and psychological well-being. Therefore, old age can be considered as the review and evaluation period and time of growth and prosperity and calm period in the remaining part of life. On the other hand, due to negative schemas for old age, these studies can be effective in changing the attitude of the community and in the promotion of mental health and hope for the future in different periods of life.

\section{Ethical Considerations}

\section{Compliance with ethical guidelines}

The ethics committee of Kharazmi University has confirmed this article.

\section{Funding}

This research did not receive any specific grant from funding agencies in the public, commercial, or not-forprofit sectors.

\section{Conflict of interest}

The authors declare no conflict of interest.

\section{Acknowledgements}

We appreciate the cooperation of all professors of Kharazmi University and all the people who participated in this research. 


\title{
بررسى معناى زندَّى و بهزيستى روانشناختى، جوانان، بزرّخسالان و سالمندان (مطالعه مقايسهاى از نظر سن)
}

\author{
"سهيلا اورنك'، هادى هاشمى رزينى'، مريم رامشينى"
}

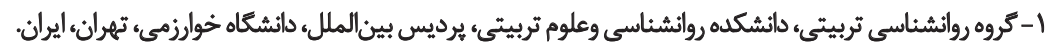

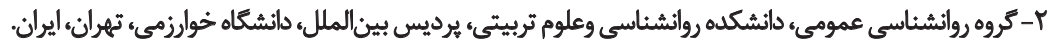

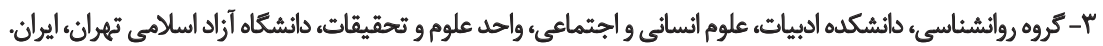

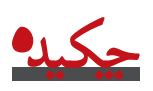

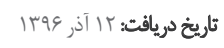

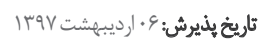

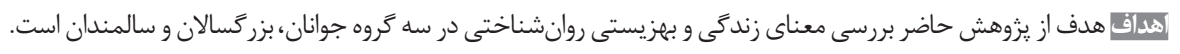

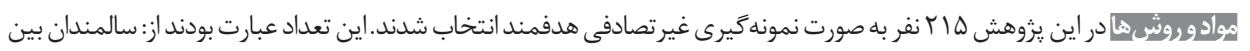

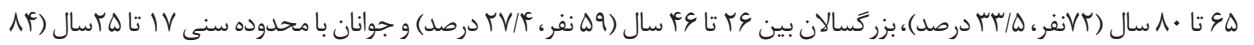

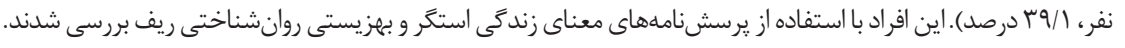

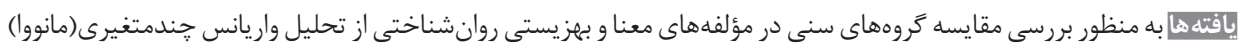

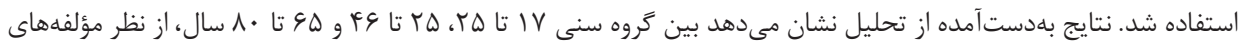

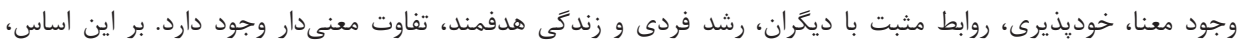

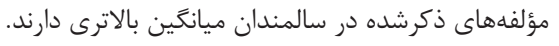

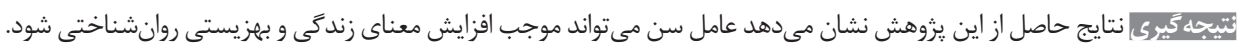

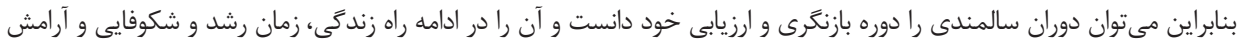

كليدوازوهان

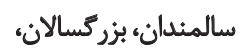
جوانانه معناي زندئيء بزيستى روانشناختي
\end{abstract}

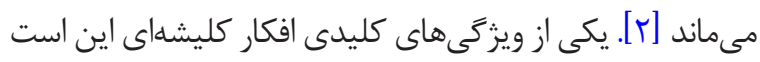

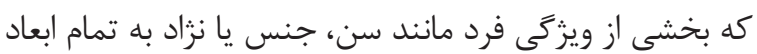

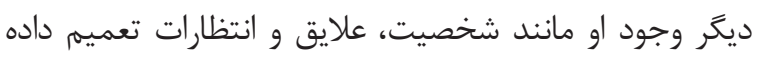

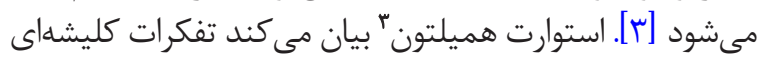

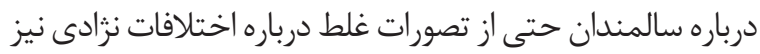

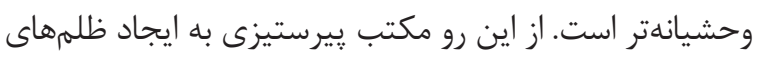

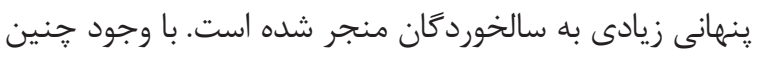

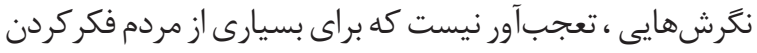

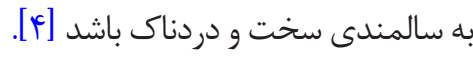

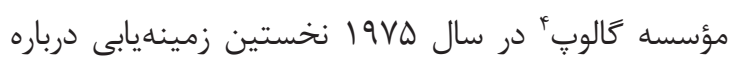

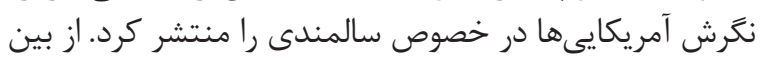
FrAF

مطالعه سالمندى را نمىتوان از باقى حيات جدا كرد، زيرا

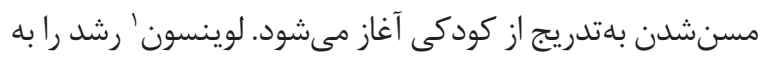

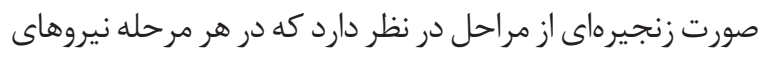

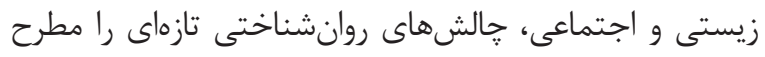

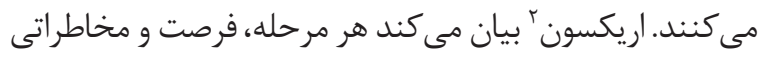

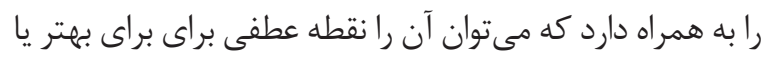
بدتر شدن دانست [1 إن. با اين حال، به نظر مىرسد در دوران سالمندى حركت در در

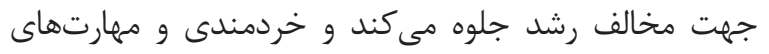

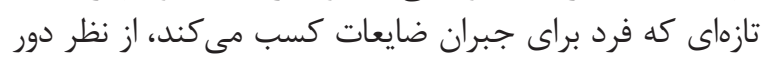

2. Ericsson 
مهمهرين مؤلفه بهزيستى روانشناختى است و وجود معنا در

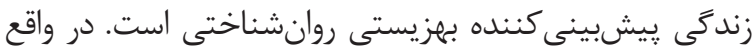

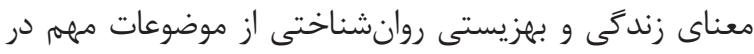

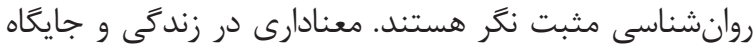

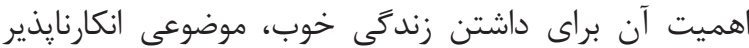

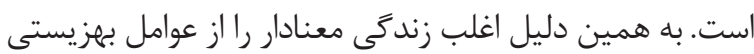

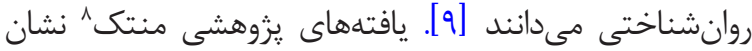

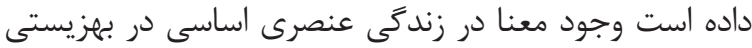

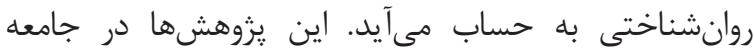

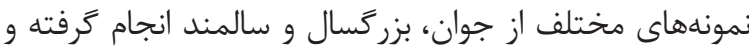

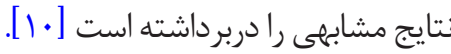
مفهوم معناى زندگى بر سه بايه استوار است: آزادى اراده،

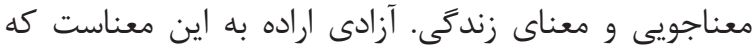

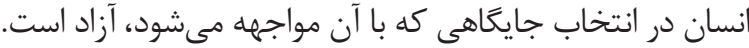

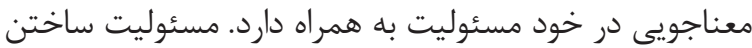

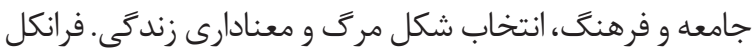

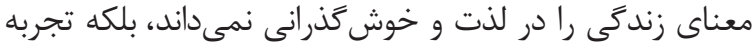

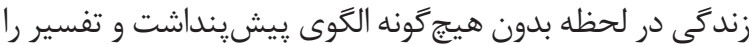

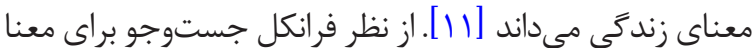

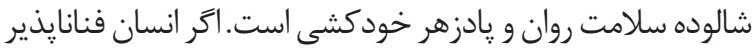

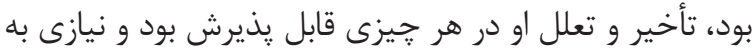

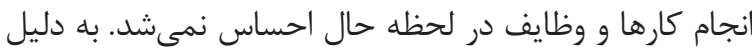

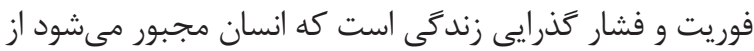

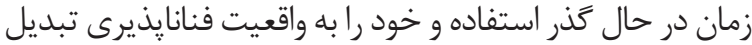

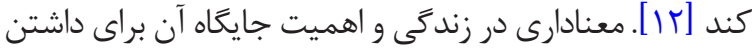

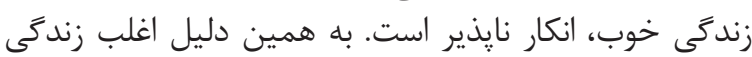

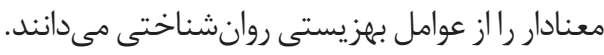

مطالعات دوروتا كدلا" و همكاران نشان داد معناى زندكى

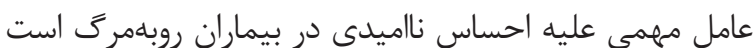

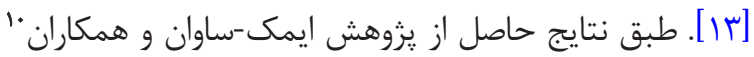

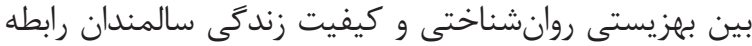

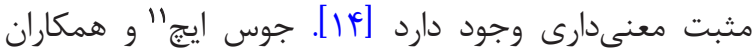

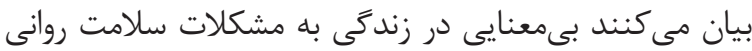

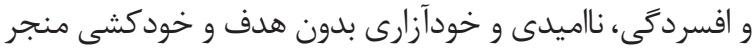

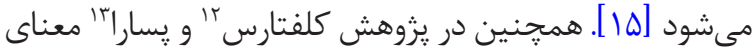

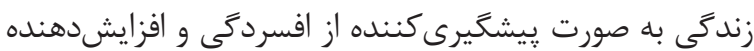

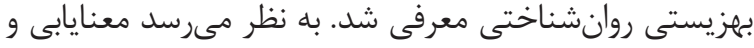

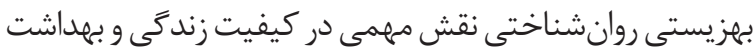

\section{Mantak Yen}

9. Dorothea kudla

10.Emek-Savas

11. Jose

12. Kleftaras

13. Psarra
سال هاى عمر قلمداد كردند و يكسوم :اسخدهند إندان سالمندى

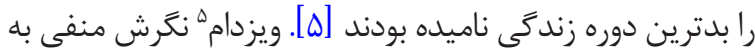

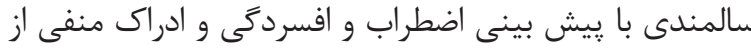

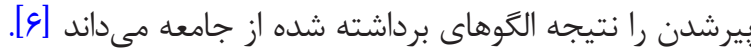

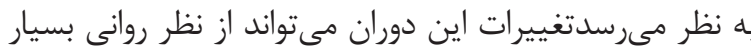

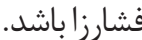

ويتوريو كايدانو" عقيده دارد تغيير بدون دخالت هيجانات

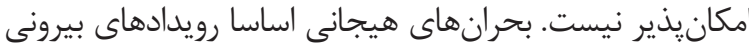

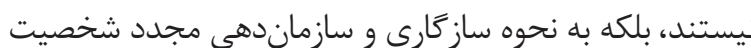

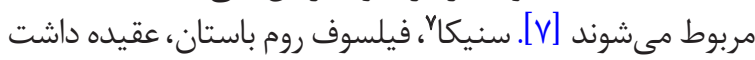

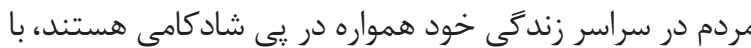

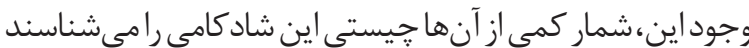

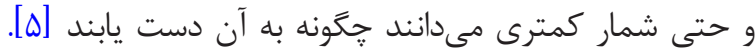

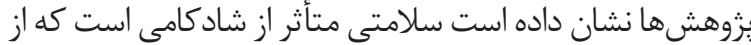

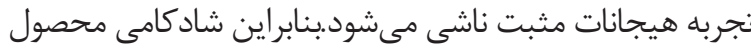

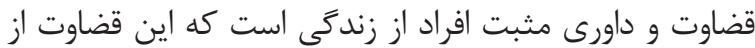

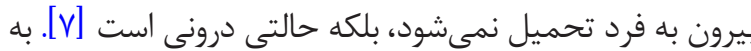

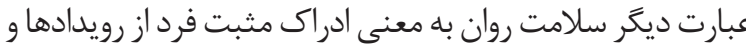

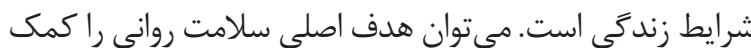

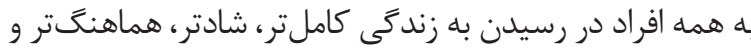

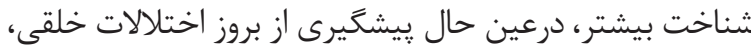
عاطفى و رفتارى دانست.

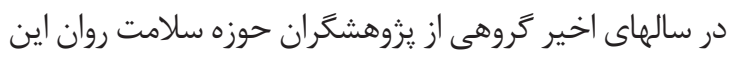

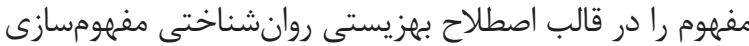

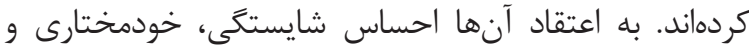

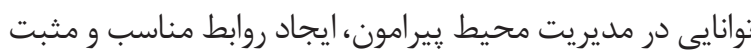

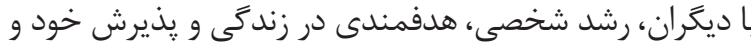

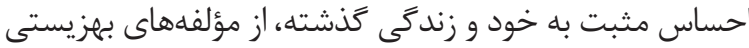

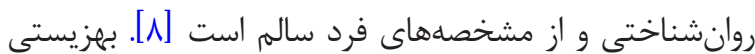

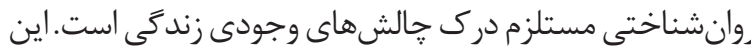

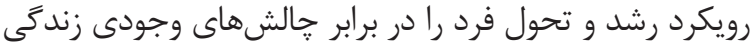

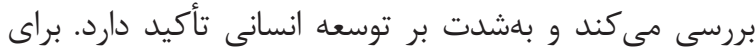

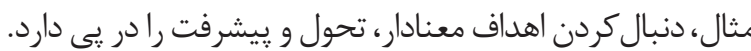

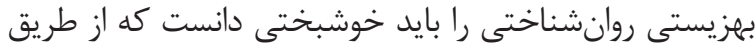

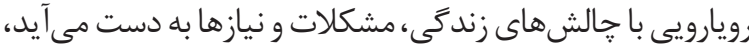

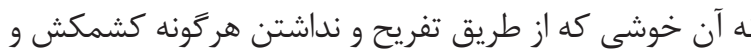

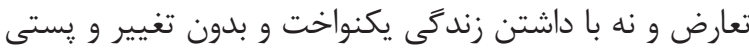

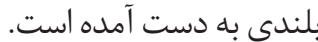

انسان تنها مخلوقى است كه از معناى مرك آكاه است و

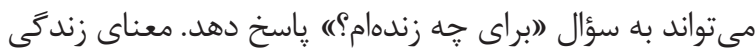


نوع يزوهشى است. كميته دانشكاه خوارزمى اين مقاله را تأييد

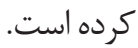

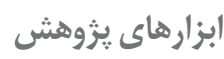

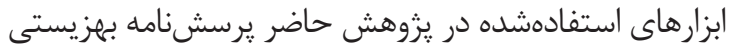

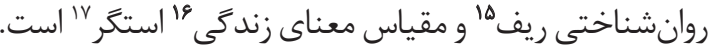

ريف مقياس بهزيستى روانشناختى ريف را تهيه كرده است

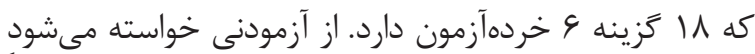

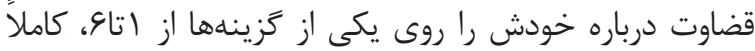

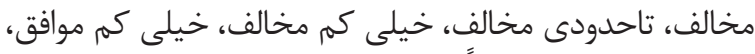

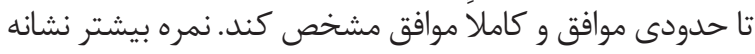

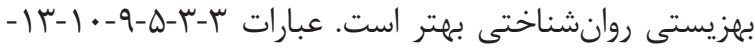

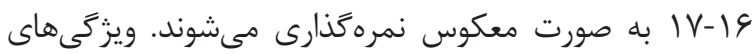

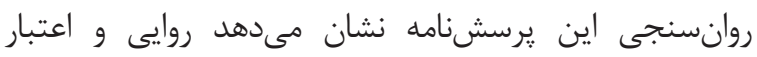

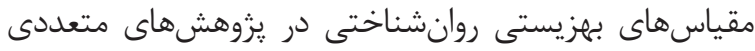

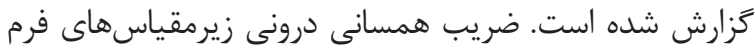

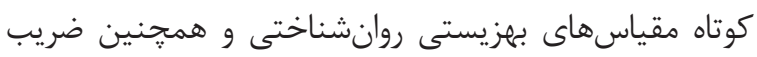

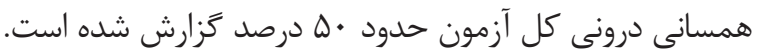

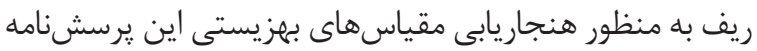

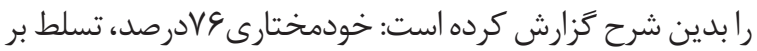

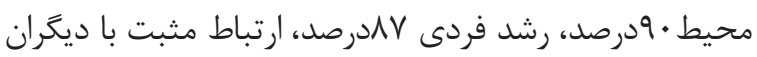

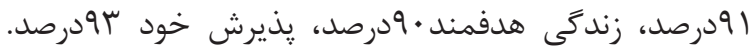

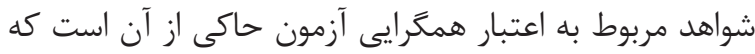

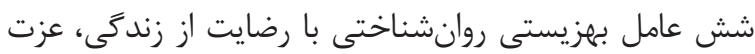

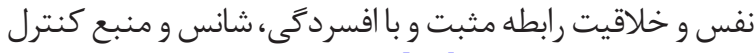

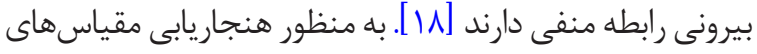

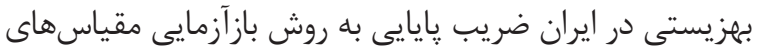

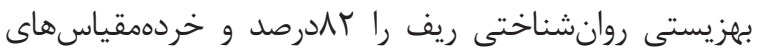

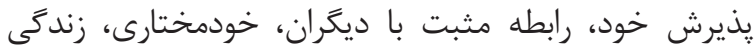

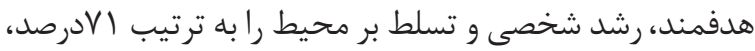

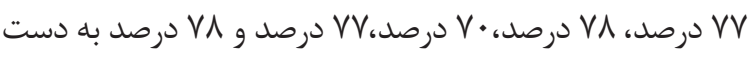

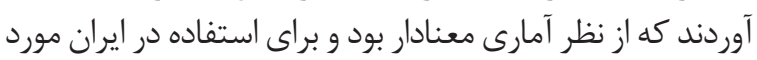

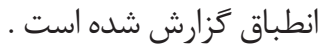

يرسشنامه معناى زندگى استخر و همكاران براى دو دو عامل

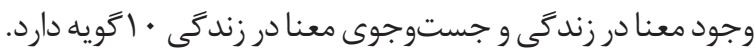

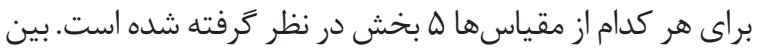

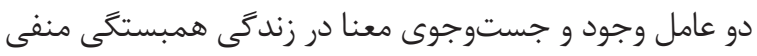

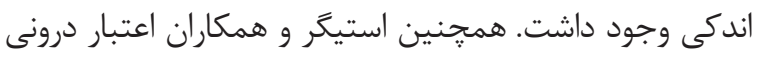

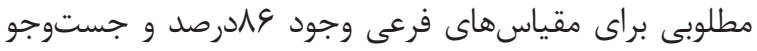

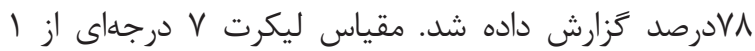

15.Ryff's Psychological Well-being Scales

16.Meaning in life Questionnaire

17. Steger
روانى در دوران سالمندى ايفا مى كنند [91]].

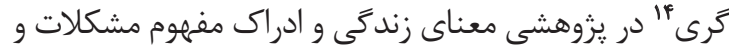

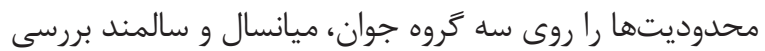

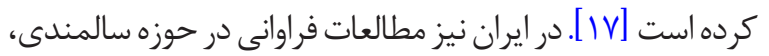

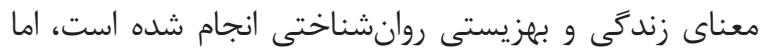

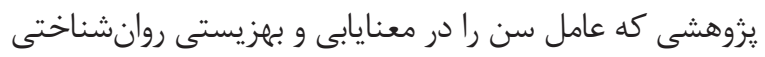

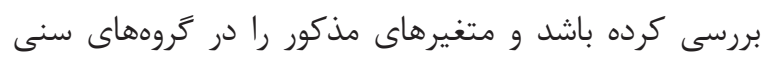

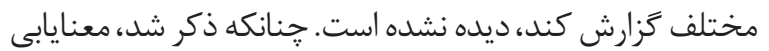

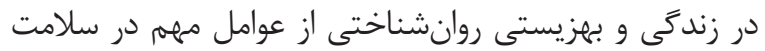

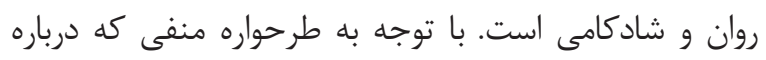

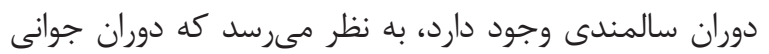

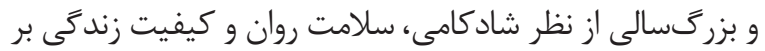

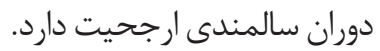

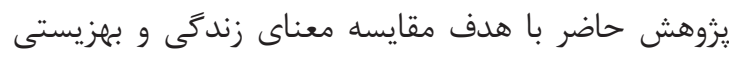

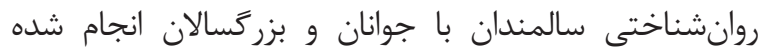

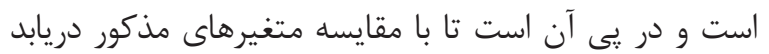

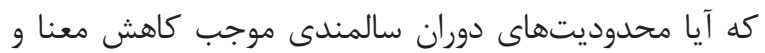

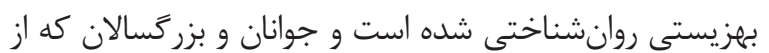

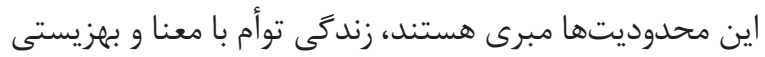

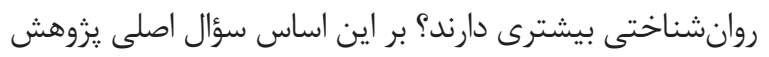

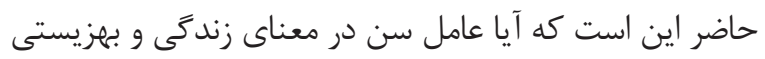
روانشناختى افراد در دورههاى مختلف سنى نقش دارئ دارد؟

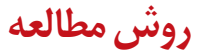

يزروهش حاضر از نوع علّمىمقايسهاى است و براى تحليل دادهها

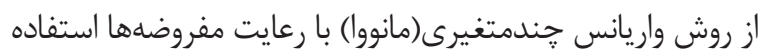

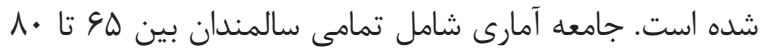

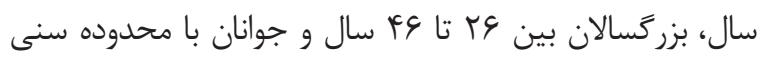

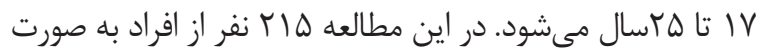

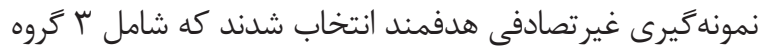

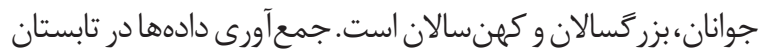

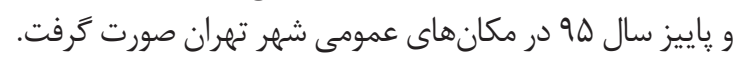

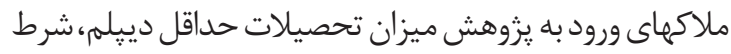

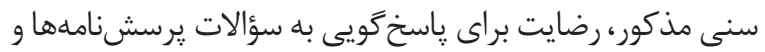

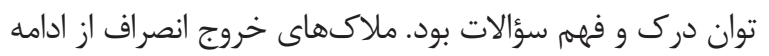

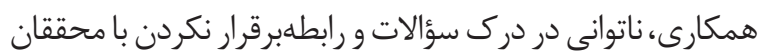

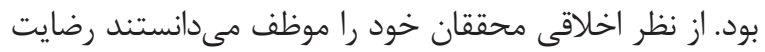

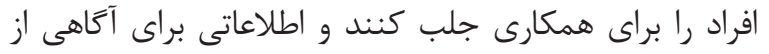

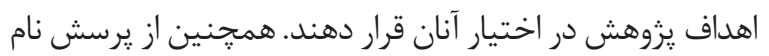

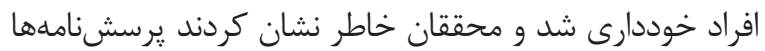

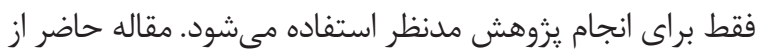

14. Gary 
مانووا، آزمون لون 19 است. نتايج آزمون لون نشان مى مدهد سطح

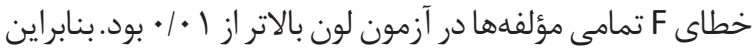

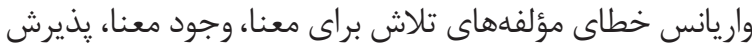

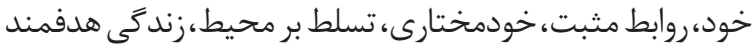
و رشد فردى در بين كروهها تفاوت معنادارى نداشتنتئ.

يكى ديكر از بيشفرضهاى مانووا، يكسانى ماتريسهاى

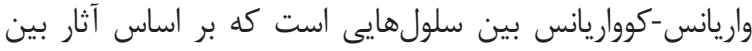

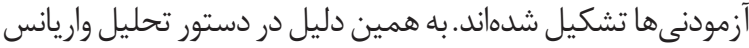

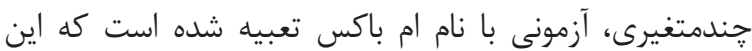

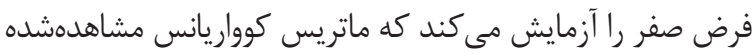

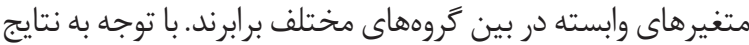

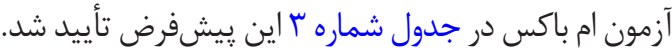
همانطور كه جدول شماره \& نشان مىدهد، نتايج آزمون

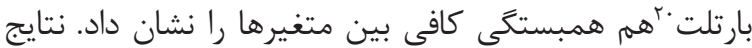

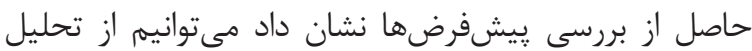

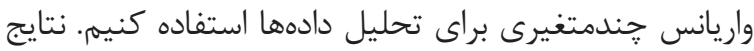

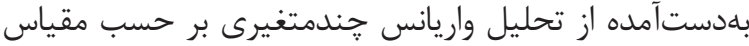

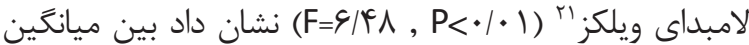

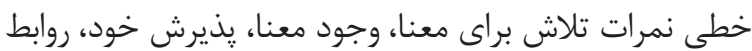

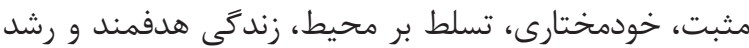

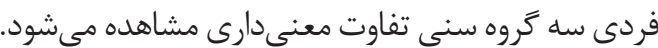

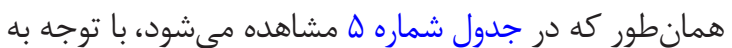

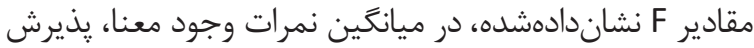

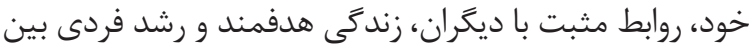

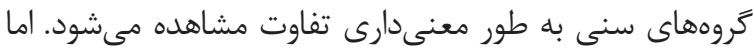

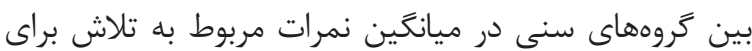

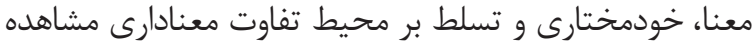

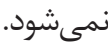

با توجه به معنىدارى نتايج تحليل واريانس براى برخى از

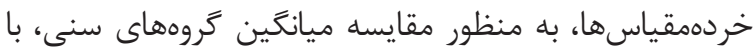

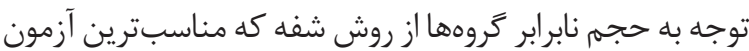
تعقيبى است، استفاده شد.

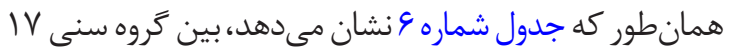

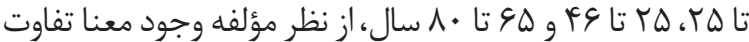

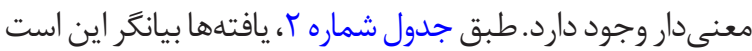

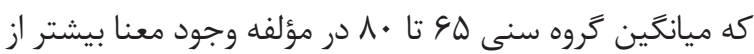

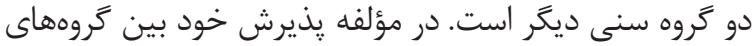

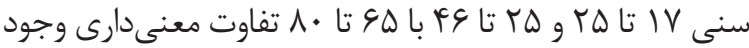

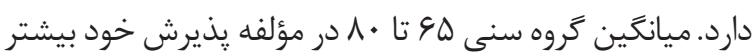

19. Levene's Test

20. Bartlet

21. Wilks's Lambda

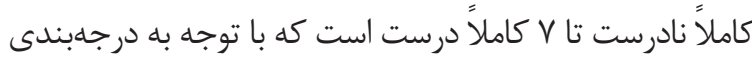

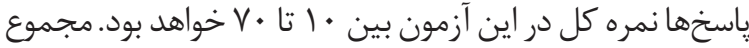

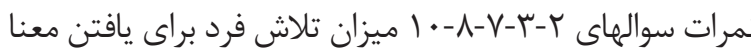

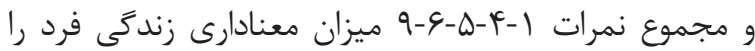

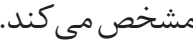

طبق يزوهشهاى استخر و همكاران اعتبار اين مقياس براى

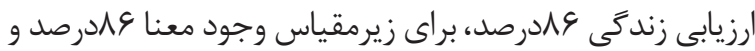

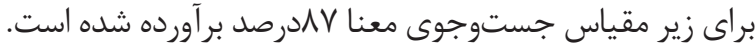

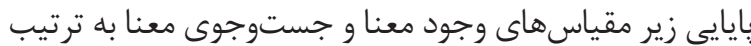

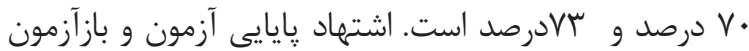

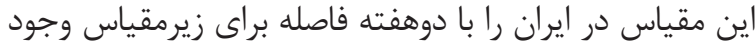

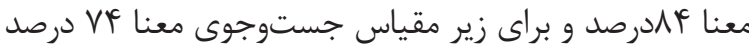

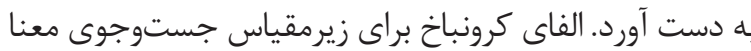

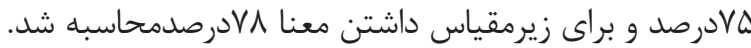

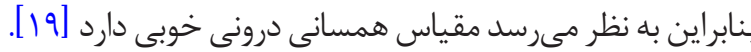

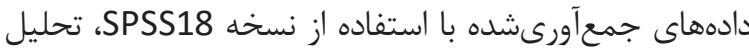

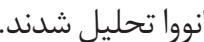

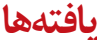

براى آزمون فرضيه مدنظر با توجه به تعداد متغيرهاى مربوط

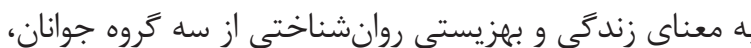

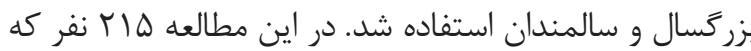

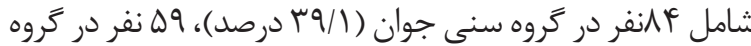

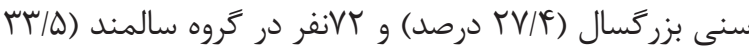

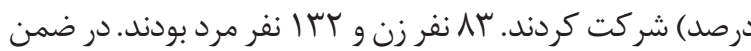

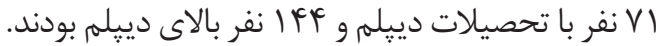

هدف اصلى اين مطالعه ياسخ به اين سؤال بود كه آيا عامل

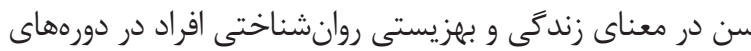

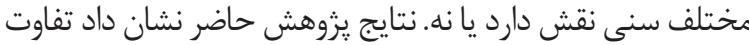

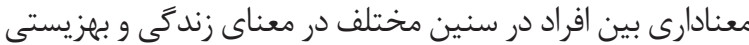

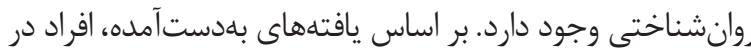

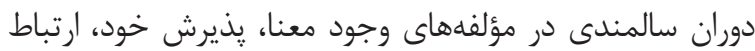

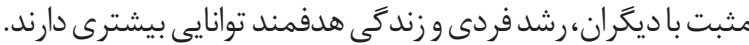
به منظور بررسى مقايسه كرومهاى سنى در مؤلفههاى معنا و

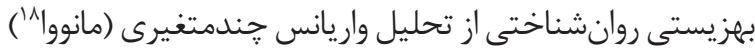

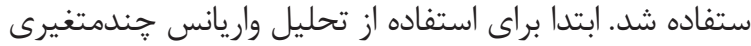

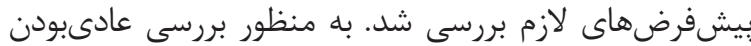

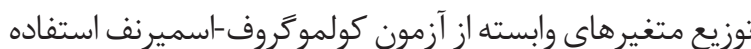

$$
\text { شده است (جدول شماره (). }
$$

همانطور كه نتايج جدول شماره r نشان مى دهدد، متغيرهاى

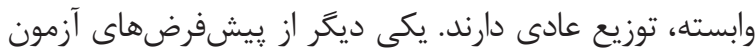

18. MANOVA 
جدول ا. نتايج آزمون كولموكروف-اسميرنف

\begin{tabular}{|c|c|c|}
\hline سطح معنىدارى & $\mathrm{K}-\mathrm{S}$ & متغير وابسته \\
\hline.$/ . \cdot 1$ & $r /$. & تلاش براى معنا \\
\hline.$/ . .1$ & $r / F \Delta$ & وجود معنا \\
\hline $.1 . .1$ & T/M & يذيرش خود \\
\hline.$/ . .1$ & $1 / I V$ & روابط مثبت \\
\hline . $/ \cdot \mathrm{HC}^{2}$ & $1 / 4 q$ & خودمختارى \\
\hline .1 .11 & V $/ \Delta$ & تسلط بر محيط \\
\hline.$/ . .1$ & $1 / r$. & زندگى هدفمند \\
\hline.$/ . .1$ & $r / 11$ & رشد فردى \\
\hline
\end{tabular}

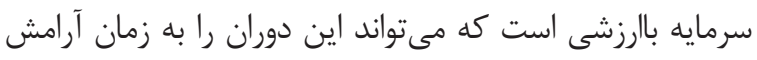

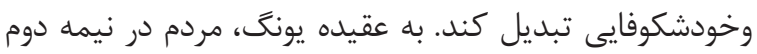

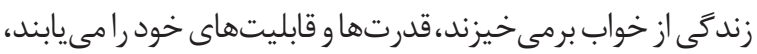

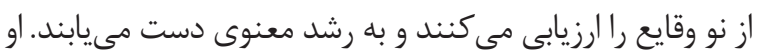

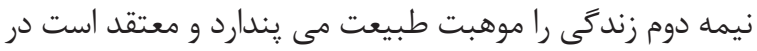

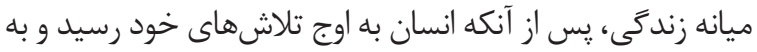

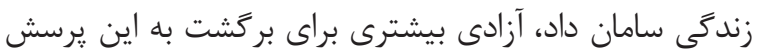

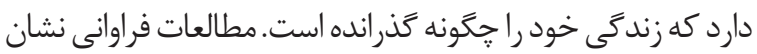

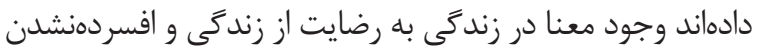

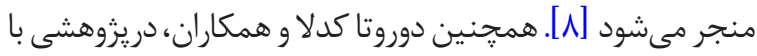

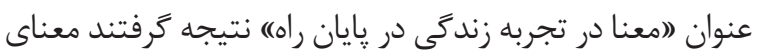

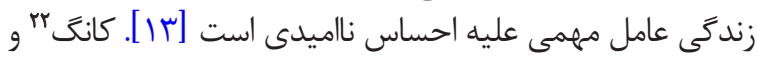

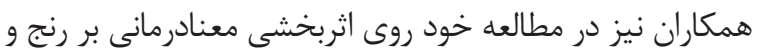

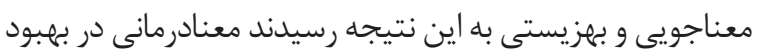

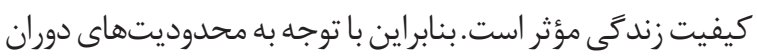

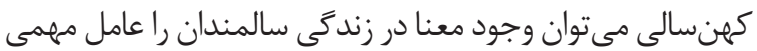

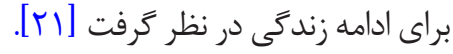

در تبيين مؤلفه يذيرش خود در دوران سالمندى، هرجند

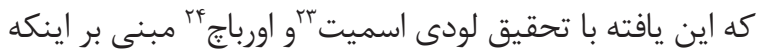

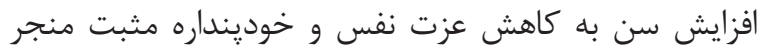

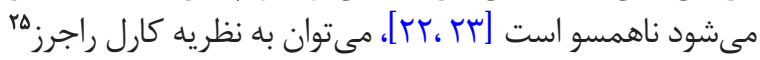

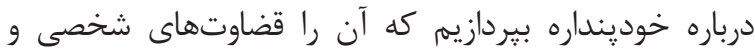

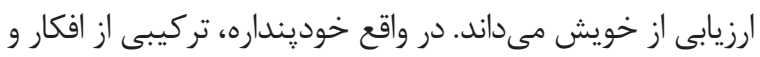

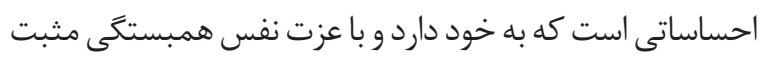

\section{Kang}

23. Lodi-Smith

24. Fujita

25.Carl Rogers

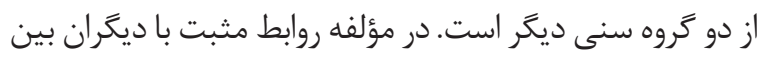

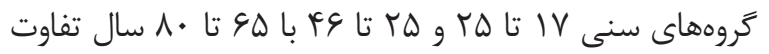

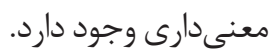

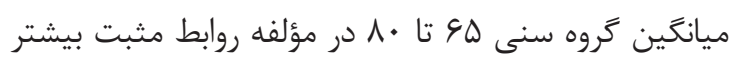

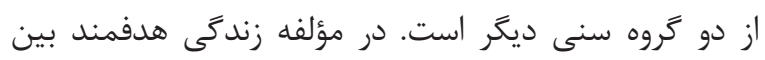

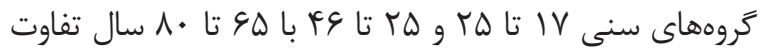

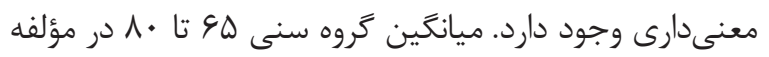

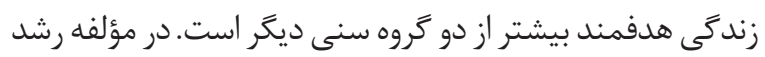

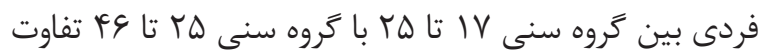

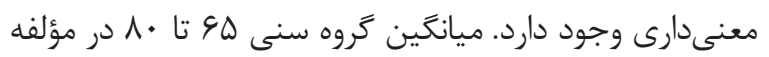

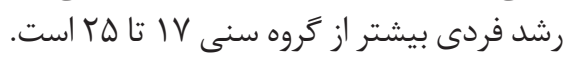

بحث

نتايج حاصل از اين يزوهش نشان داد از نظر مؤلفه وجود معنا

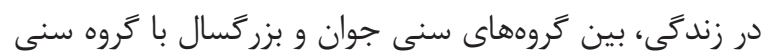

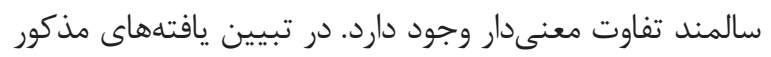

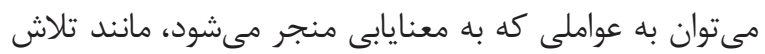

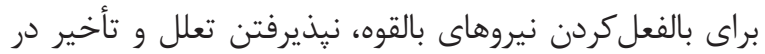

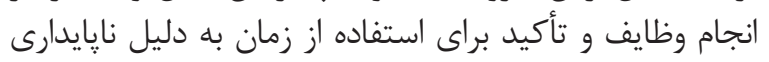

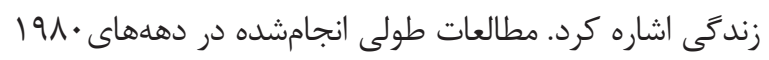

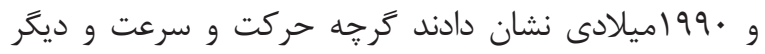

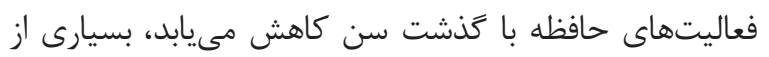

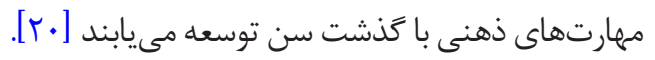
بر اين اساس به نظر مىرسد دوران سالمندى در مقايسه با ديكر ماند

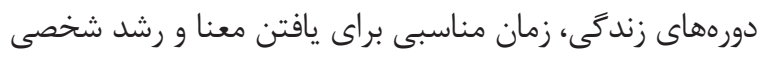

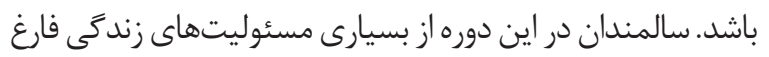

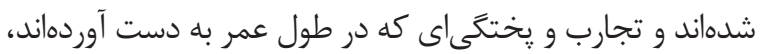


جدول ז. ميانگين و انحراف استاندارد

\begin{tabular}{|c|c|c|c|}
\hline انحراف استاندارد & ميانتين & سن & مؤلفهها \\
\hline S/rVT & TA/TYT. & $I V-r \Delta$ & \\
\hline$r / 909$ & tr/Arq & teqE & تلاش براى معنا \\
\hline V/Ag & TV/\&AY & $\Leftrightarrow \Delta-\Lambda$. & \\
\hline$r / 991$ & 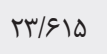 & $I V-r \Delta$ & \\
\hline$\Gamma / \Delta V \Delta$ & TM/lQ. & عe-re & وجود معنا \\
\hline $11 / \Delta 1 F^{F}$ & $r V / \cdots$ & $\varphi_{\Delta-\Lambda .}$ & \\
\hline $1 / 9 \cdot 4$ & $11 / \Delta \Delta 1$ & $I V-r \Delta$ & \\
\hline $1 / 9 \cdot \Delta$ & IT/IMT & & يذيرش خود \\
\hline r/IVD & $1 \% / F \Delta$. & $\Leftrightarrow \Delta-\Lambda$. & \\
\hline אזr/ז & N/TqL & $I V-r \Delta$ & \\
\hline T/TFV & Q/TEY & rq-re & روابط مثبت با ديكران \\
\hline$r / G 49$ & $\mid r / \mu$. & $9 \Delta-\Lambda$. & \\
\hline$r / f \mid f$ & $\mid r / r \cdot \Delta$ & $I V-r \Delta$ & \\
\hline r/meq & IT/FME & & خودمختارى \\
\hline$r / V T \Delta$ & $I T / \Lambda \Delta$. & $\Leftrightarrow \Delta-\Lambda$. & \\
\hline ט.r. & $1 \pi / 1 \cdot r$ & $I V-r \Delta$ & \\
\hline $1 / N$ & $1 \% / 9 \cdot 0$ & re-re & تسلط بر محيط \\
\hline$r / r$. & $1 \% / \Delta \Delta$. & $\varphi \Delta-\Lambda$. & \\
\hline$\Gamma / \Delta 1 \Delta$ & $9 / 197$ & $I V-r \Delta$ & \\
\hline T/AVT & 1.1 .11 & & زندحى هدفمند \\
\hline$\Gamma / \cdot \Lambda \Delta$ & IT/KGG & $\Leftrightarrow \Delta-\Lambda$. & \\
\hline $1 / 901$ & $\mid r / F F A$ & $I V-r \Delta$ & \\
\hline $1 / v \cdot r$ & IT/FME & rq-rq & رشد فردى \\
\hline V/Vrq & $\mid f / F 98$ & $9 \Delta-\Lambda$. & \\
\hline
\end{tabular}

خويش آكًاه مىشود، با نزديكشدن به اين احساسات فرصت

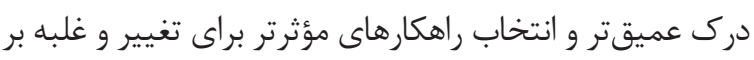

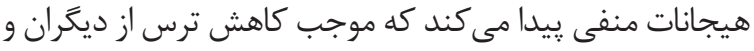

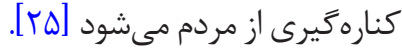
همجنين نف "و همكاران بيان كردند شفقت به خود به طور 27. Neff
دارد [rr]. از سوى ديگر ارتباط با خويش و خودينداره مثبت

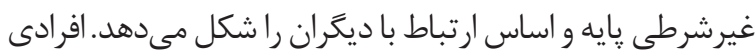

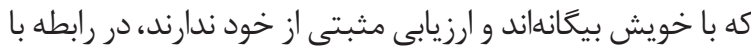

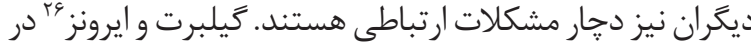

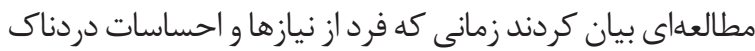

26. Gilbert \& Irons

جدول r. نتايج آزمون ام. باكس

\begin{tabular}{|c|c|c|}
\hline سطح معنى دارى & $\mathrm{F}$ & Box's M \\
\hline . $/ Q T$ & $\Delta / / \zeta$ & rqs/vq \\
\hline
\end{tabular}

d 


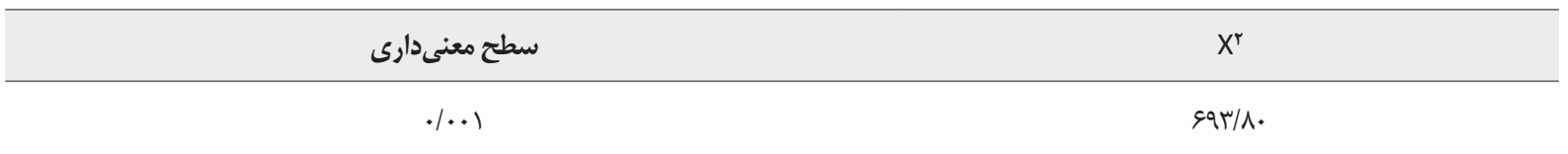

ic

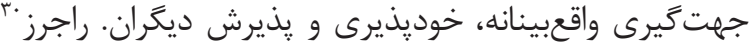

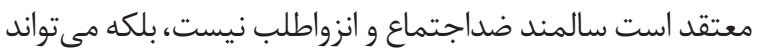

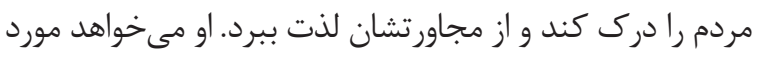

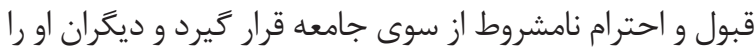

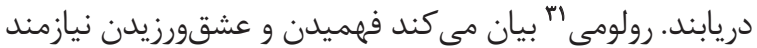

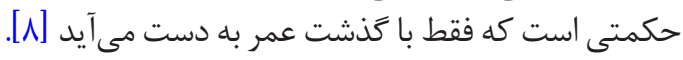
طبق نتايج حاصل از يزوهش ايمك-ساوان و همكاران بين

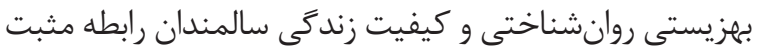

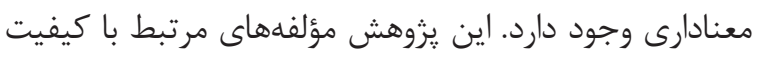

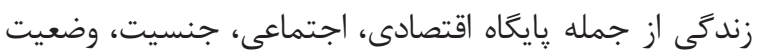

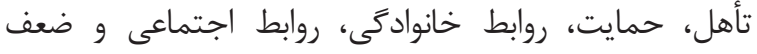
جسمانى، سن و نكرش مثبت به سالمندى و امكانات رفاهى رابط را رابط

30. Rogers

31.Rollo May

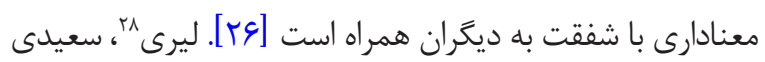

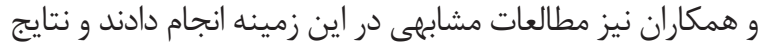

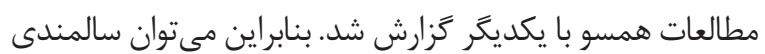

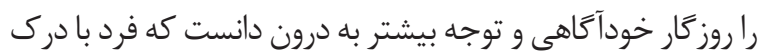

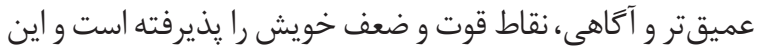

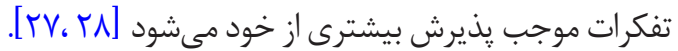

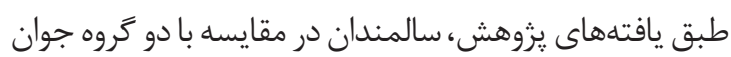

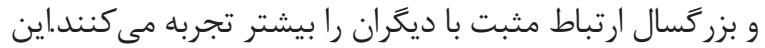

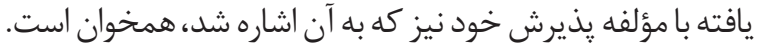

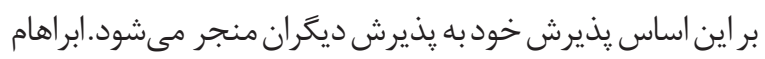

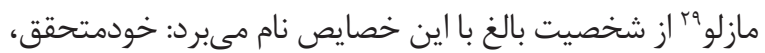

28. Leary

29. Maslow

جدول ه. اثرات بين آزمودنى در مؤلفههاى معناو بهزيستى روان شناختى

\begin{tabular}{|c|c|c|c|c|c|c|}
\hline معنىدارى & $\mathbf{F}$ & ميانكين مجذورات & درجه آزادى & مجموع مجذورات & متغير وابسته & منبع تغيير \\
\hline -/AME & . /NAY & $V / \varepsilon+\Lambda$ & r & $|Q / T| S$ & تلاش براى معنا & \multirow{8}{*}{ گروه (سن) } \\
\hline .1 .99 & D/TQF & TE9/.IT & r & & وجود معنا & \\
\hline $.1 .+1$ & سות/11 & GT/TrV & r & $\mid \pi \Psi / \Delta \Delta \Delta$ & يذيرش خود & \\
\hline.$/ .+1$ & TY/TIV & $r \Lambda \mu / F I F$ & r & $\Delta S g / A T \Lambda$ & روابط مثبت & \\
\hline$\cdot / F \Delta \Delta$ & $\cdot / \mathrm{vq}$ & $\digamma / 9 \Delta 1$ & r & $9 / 9.1$ & خودمختارى & \\
\hline.$/|n|$ & $\mid / V \wedge I$ & $1 . / F \wedge S$ & r & $r . / 9 r 1$ & تسلط بر محيط & \\
\hline $.1 .+1$ & $15 / 119$ & $11 . / 99$. & r & TrI/QR. & زندكَى هدفمند & \\
\hline \multirow[t]{9}{*}{.$/ \cdot r$. } & r/q9 & $\Lambda F / r \Lambda V$ & r & IFN/DVD & رشد فردى & \\
\hline & & FI/NGS & $M$ & $V A V \cdot / V q T$ & تلاش براى معنا & \multirow{8}{*}{ خطا } \\
\hline & & Q./GML & $M$ & $9 D 19 / T \Delta F^{\circ}$ & وجود معنا & \\
\hline & & س س & $M$ & D.N/Tr. & يذيرش خود & \\
\hline & & $11 / \& \Delta \Delta$ & $M$ & $r \mid l q / 1 r$. & روابط مثبت & \\
\hline & & אצ & $M$ & IIW/rAV & خودمختارى & \\
\hline & & D/N\&S & $M$ & $11.9 / \Delta \Delta \Lambda$ & تسلط بر محيط & \\
\hline & & $V / N \mid{ }^{\prime}$ & $M$ & IfएW/A94 & زندگى هدفمند & \\
\hline & & rM/Ir & $M$ & rasq/rev & رشد فردى & \\
\hline
\end{tabular}


جدول 9. نتايج آزمون تعقيبى شفه

\begin{tabular}{|c|c|c|c|c|c|}
\hline سطح معنىدارى & خطاى استاندارد & اختلاف ميانكين & سن & سن & مؤلفهها \\
\hline . /qयू & | I I & . Isefte & re-te & $I V-r \Delta$ & \multirow{6}{*}{ و وجود معنا } \\
\hline אז./. & $|/ K T|$ & G & $\varepsilon_{\Delta-\Lambda .}$ & & \\
\hline (9TD & 1/T\&G & - orete & $I V-r \Delta$ & GE-Fe & \\
\hline$\cdot 1 \cdot 11$ & $\mid / M+1$ & $-\Upsilon / \wedge 9 १ 1$ & $\varphi_{\Delta-\Lambda}$. & & \\
\hline אז./. & I/TKI & T/rNFE & $I V-r \Delta$ & $\varphi_{\Delta-\Lambda .}$ & \\
\hline$\cdot 1 \cdot 11$ & $|/ M F|$ & $r / \wedge<q 1$ & re-te & & \\
\hline . & . & $-\bullet / \Delta M$ & re-te & $I V-r \Delta$ & \multirow{6}{*}{ يذيرش خود } \\
\hline.$/ .+1$ & ./ тqv8 & $-1 / \wedge q \wedge \vee$ & $\varsigma_{\Delta-\Lambda .}$ & & \\
\hline . & . & $\cdot \mid \Delta \Lambda \cdot \Lambda$ & $I V-r \Delta$ & TE-TE & \\
\hline.$/ .14$ & DצF & $-1 /$ TIVq & $\varsigma_{\Delta-\Lambda .}$ & & \\
\hline.$/ .1$ &.$/$ T9v8 & ।/А९४マ & $I V-r \Delta$ & $\varepsilon \Delta-\Lambda$. & \\
\hline .1 .14 & . & 1/rivg & re-re & & \\
\hline . & $\cdot 19 \cdot W$ & $-\cdot / 9994$ & re-te & $I V-r \Delta$ & \multirow{6}{*}{ روابط مثبت با ديخران } \\
\hline $.1 .+1$ & . IDNET & $-4 / \cdot \cdot \Delta 1$ & $\Leftrightarrow \Delta-\Lambda$. & & \\
\hline - & $\cdot 19 \cdot W$ & 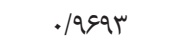 & $I V-r \Delta$ & te-te & \\
\hline $.1 \cdot .1$ & . Iatra & $-r / \cdot r \Delta \Lambda$ & $\varepsilon_{\Delta-\Lambda .}$ & & \\
\hline $.1 .+1$ & . IDAST & $\varphi / \cdot \Delta \mid$ & $I V-r \Delta$ & $\varepsilon \Delta-\Lambda$. & \\
\hline $.1+.1$ & . Iatra & r/. & re-te & & \\
\hline.$/ 971$ &.$/ 49911$ & $-\cdot \mid I T I F$ & re-re & $I V-r Q$ & \multirow{6}{*}{ زندگى هدفمند } \\
\hline.$/ .+1$ &.$|\& \lambda| \notin \varepsilon$ & -r/rצqr & $\varsigma \Delta-\Lambda$. & & \\
\hline.$/ 971$ &.$/ 49911$ & ./KIF & $I V-r \Delta$ & $r \varepsilon-r q$ & \\
\hline.$/ .+1$ & . & - T/TYVA & $\varsigma_{\Delta-\Lambda .}$ & & \\
\hline.$/ .+1$ & $. / \& \lambda \mid \& s$ & T/Kदar & $I V-r \Delta$ & $9 \Delta-\Lambda$. & \\
\hline.$/ .1$ & . IATADL & T/MFVA & Fq-F| & & \\
\hline $1 / \ldots$ & ./AVIVq &.$/ \cdot 14 \lambda$ & TE-Yq & $I V-r \Delta$ & \multirow{6}{*}{ رشد فردى } \\
\hline$+1+4$ & ./v^я. & $-r / \cdot 1 V q$ & $\varepsilon \Delta-\Lambda$. & & \\
\hline $1 / \cdots$ & $\cdot / 11 v 9$ & $-.1 \cdot 14 \lambda$ & $I V-r \Delta$ & TE-Fq & \\
\hline .1 .99 & . IN\&IV & $-\Gamma / \cdot r T V$ & $\varepsilon_{\Delta}-\lambda$. & & \\
\hline $.1 \cdot 4$. & ./ ২ム৭. & $r / \cdot 1 v q$ & $I V-r \Delta$ & $\Leftrightarrow \Delta-\lambda$. & \\
\hline .1 .99 & . & T/FTr & TE-Yq & & \\
\hline
\end{tabular}


زندگى جلوهَ مىشود [بس]. در اين زمينه يزوهشهايى انجام

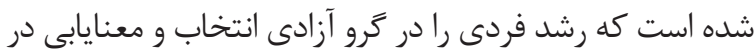

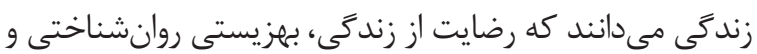

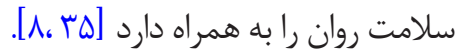

\section{نتيجه تيرى نهايى}

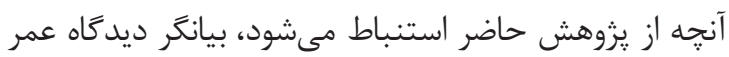

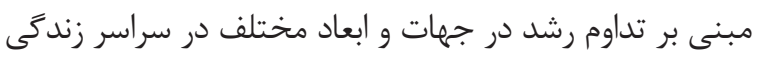

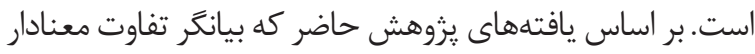

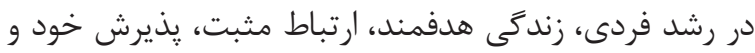

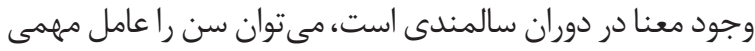

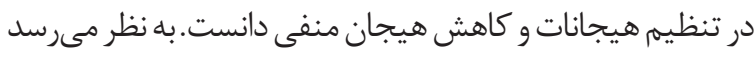

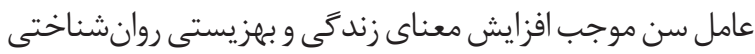

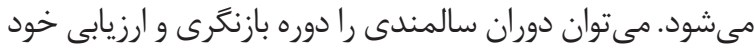

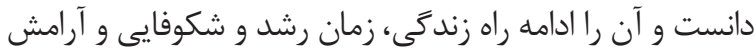

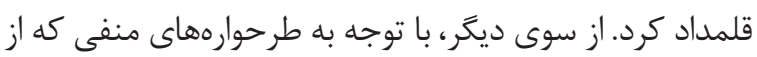

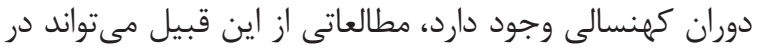

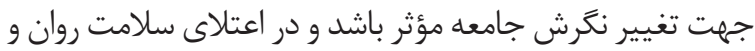

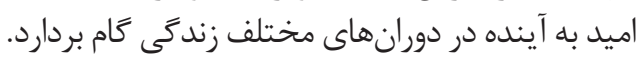

از محدوديت هاى يزوهش حاضر مى توان به نبود مطالعات مشابه

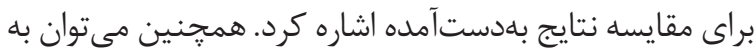

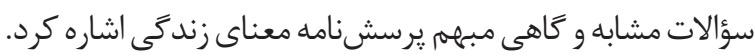

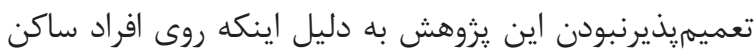

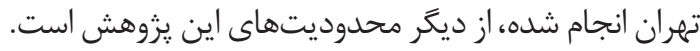

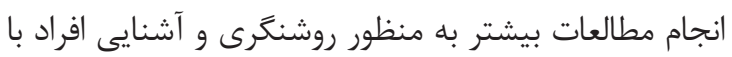

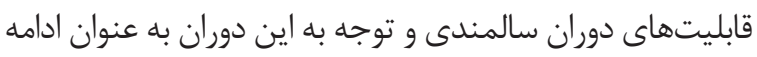

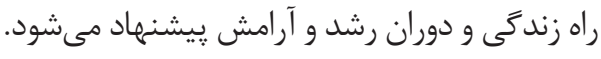

$$
\text { تشكر و قدردانى }
$$

در زايان، از همكارى و مساعدت استادان محترم دانشعاه

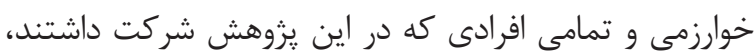
كمال تشكر و قدردانى مى شود. اين مقاله حامى مالى نداردي
بررسى مى كند و بهزيستى روانشناختى را از عوامل تأثير كذار

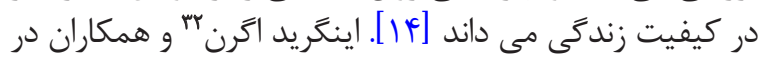

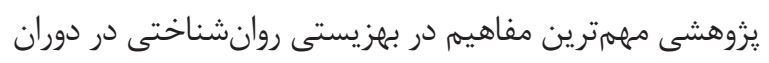

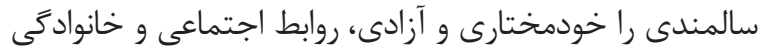

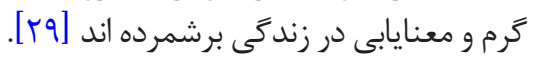

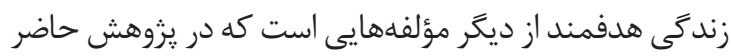

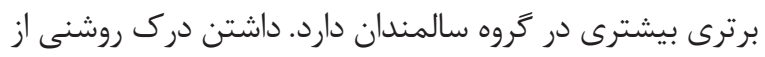

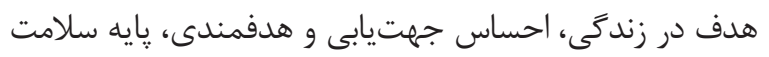

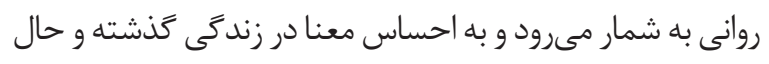

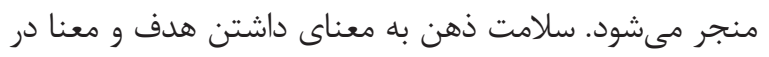

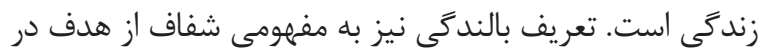

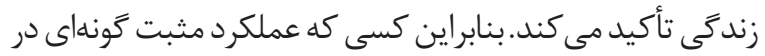

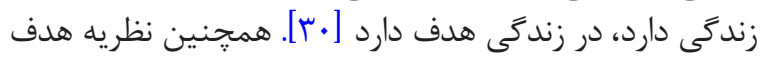

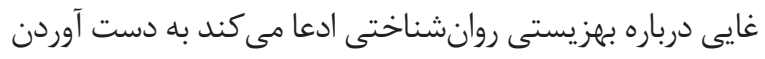

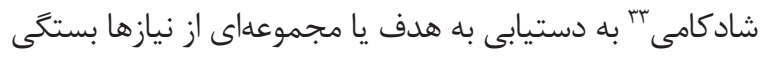

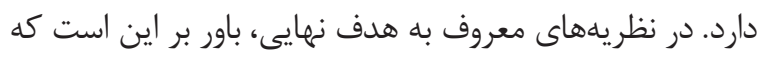

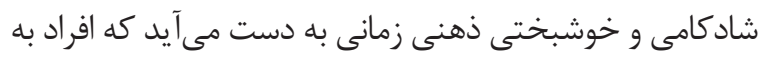

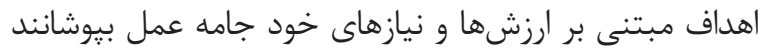

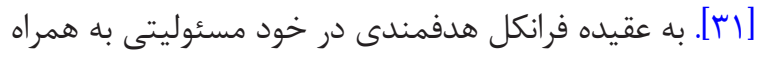

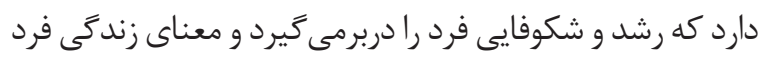

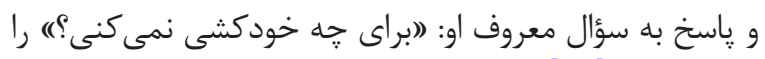

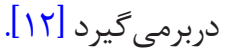

رولومى عقيده دارد كسى كه بتواند با بالاترين ارزشهايش دردي

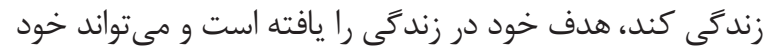

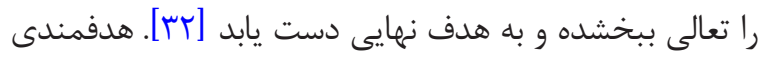

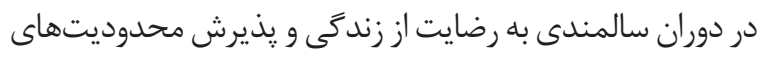

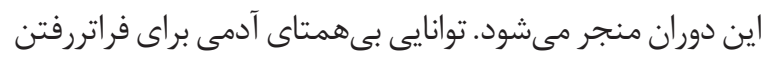

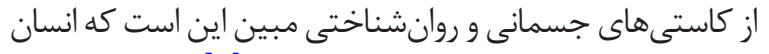

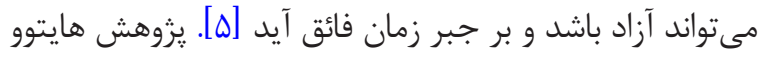

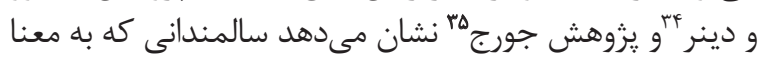

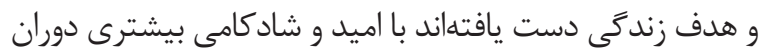

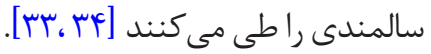
بر اين اساس رشد فردى رانمىتوان جدا از مؤلفههاى ذكرشده

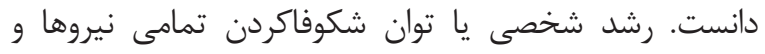

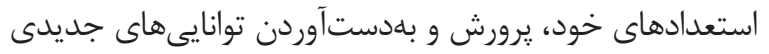

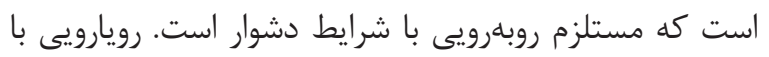

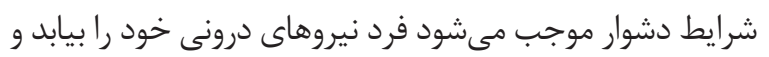

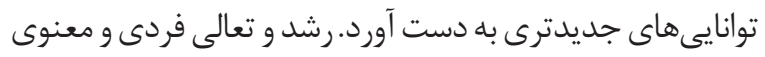

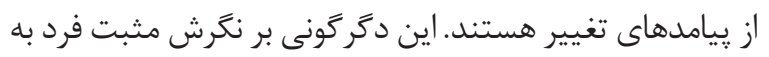




\section{References}

[1] Berk L. Development through the lifespan [Y. Sayed Mohammadi Persian trans]. Tehran: Arasbaran; 2007.

[2] Walsh S. Conducting research with the elderly: ethical concerns for a vulnerable population. Southern Online Journal of Nursing Research. 9(4).

[3] Levy BR, Pilver CE, Pietrzak RH. Lower prevalence of psychiatric conditions when negative age stereotypes are resisted. Social Science \& Medicine. 2014; 119:170-4. [DOI:10.1016/j. socscimed.2014.06.046]

[4] Macintyer A, Atval A. Occupational therapy and older people [M. Shaterian, M. Hoseini, Persian trans]. Tehran: Danjeh; 2011.

[5] Guttmann D. Finding meaning in life, at midlife and beyond [N. Ghasemi Persian trans]. Shiraz: Navid-e Shiraz; 2008.

[6] Visdom N. Social acceptance of discrimination [PhD Dessertation]. Oklahoma: Oklahoma State University; 2010

[7] Lazarus R. Coping with aging [J. Najafi Zand Persian trans]. Tehran: Danjeh; 2016.

[8] Orang S. [The relationship between early maladaptive schemas with meaning of life and well-being elderly (Persian)] [MA thesis]. Tehran: Kharazmi University; 2016.

[9] Doğan T, Sapmaz F, Tel FD, Sapmaz S, Temizel S. Meaning in Life and Subjective Well-Being among Turkish University Students. Procedia - Social and Behavioral Sciences. 2012; 55:612-7. [DOI:10.1016/j.sbspro.2012.09.543]

[10] Yuen M, Yau J. Relation of career adaptability to meaning in life and connectedness among adolescents in Hong Kong. Journal of Vocational Behavior. 2015; 91:147-56. [DOI:10.1016/j.jvb.2015.10.003]

[11] Frankl VE. The will to meaning foundations and applications of logotherapy [M. Milani Persian trans]. Tehran: Dorsa; 2014.

[12] Frankl VE. A review on existential psychology and psychotherapy [A. Mohammd Pour Persian trans]. Tehran: Dorsa; 2010.

[13] Kudla D, Kujur J, Tigga S, Tirkey P, Rai P, Fegg MJ. Meaning in life experience at the end of life: validation of the Hindi version of the schedule for meaning in life evaluation and a cross-cultural comparison between Indian and German palliative care patients. Journal of Pain and Symptom Management. 2015; 49(1):79-88. [DOI:10.1016/j.jpainsymman.2014.05.013] [PMID]

[14] Durusu Emek-Savaş D, Güntekin B, Yener GG, Başar E. Decrease of delta oscillatory responses is associated with increased age in healthy elderly. International Journal of Psychophysiology. 2014; 94(2):235. [DOI:10.1016/j.ijpsyy cho.2014.08.913]

[15] Marco JH, Garcia-Alandete J, Pérez S, Guillen V, Jorquera M, Espallargas $\mathrm{P}$, et al. Meaning in life and non-suicidal selfinjury: A follow-up study with participants with Borderline Personality Disorder. Psychiatry Research. 2015; 230(2):561-6. [DOI:10.1016/j.psychres.2015.10.004]

[16] Kleftaras G, Psarra E. Meaning in Life, Psychological Well-Being and Depressive Symptomatology: A Compara- tive Study. Psychology. 2012; 3(4):337-45. [DOI:10.4236/ psych.2012.34048]

[17] Reker GT. Meaning in life of young, middle-aged, and older adults: factorial validity, age, and gender invariance of the Personal Meaning Index (PMI). Personality and Individual Differences. 2005; 38(1):71-85. [DOI:10.1016/j.paid.2004.03.010]

[18] Bayani AA, Mohammad Koochekya A, Bayani A. [Reliability and Validity of Ryff's Psychological Well-being Scales (Persian)]. Iranian Journal of Psychiatry \& Clinical Psychology. 2008; 14(2):146-151.

[19] Eshtehad A. Investigation of group therapy on well-being student in university [MSc. thesis]. Tehran: Psychology Institution University; 2009.

[20] Asghari N. [Effects of logotherapy on depression of old people (Persian)] [MSc. thesis]. Tehran: Payam-e Noor University; 2010.

[21] Kang KA, Im JI, Kim HS, Kim SJ, Song MK, Sim S. The effect of logotherapy on the suffering, finding meaning, and spiritual well-being of adolescents with terminal cancer. Journal of Korean Academy of Child Health Nursing. 2009; 15(2):136 [DOI:10.4094/jkachn.2009.15.2.136]

[22] Orbach A. Counseling older clients [A. Khodabakhsi Koolaee Persian trans]. ( ( $^{\text {th }}$ ed). Tehran: Jungle; 2010.

[23] Lodi-Smith J, Roberts BW. Getting to Know Me: Social Role Experiences and age differences in self-concept clarity during adulthood. Journal of Personality. 2010; 78(5):1383-410 [DOI:10.1111/j.1467-6494.2010.00655.x]

[24] Orang T. [The study psychological dependency caused by chat on the internet (Persian)] [MA thesis]. Tehran: Alzahra University; 2004

[25] Gilbert P, Irons C. Focused therapies and compassionate mind training for shame and self attacking. In: P. Gilbert editor. Compassion: Conceptualisations, research and use in psychotherapy. London: Routedge; 2005.

[26] Neff KD, Pommier E. The relationship between self-compassion and other-focused concern among college undergraduates, community adults, and practicing meditators. Self and Identity. 2013; 12(2):160-76. [DOI:10.1080/15298868.2011.6495 46]

[27] Leary MR. The curse of the self. Self awareness, egotism, and the quality of human life. New York: Oxford University Press; 2004. [DOI:10.1093/acprof:oso/9780195172423.001.0001]

[28] Saeedi Z, Ghorbani N, Reza Sarafraz M, Sharifian MH. [The relationship between self-compassion, self-esteem and selfconscious emotions regulation (Persian)]. Research in Psychological Health. 2012; 6(3):1-9.

[29] Bolmsjö IÅ, Sandman L, Andersson E. Everyday ethics in the care of elderly people. Nursing Ethics. 2006; 13(3):249-63. [DOI:10.1191/0969733006ne875oa]

[30] Frankl VE. The will to meaning foundations and applications of logotherapy. New York: Plume; 2014.

[31] Diener E. What is positive about positive psychology? The curmudgeon and Pollyanna. Psychological and Social Psychology. 68:653-663; 2003. 
[32] May R. Man's search for himself [M. Sorayya Persian trans]. Tehran: Danjeh; 1994

[33] Tov W, Diener E. The well-being of nations. Linking together trust, cooperation and democracy. In B. A. Sullivan, M. Snyder editors. The Political Psychological of Effective Human Interaction; 2008.

[34] George LK. Still happy after all these years: research frontiers on subjective well-being in later life. The Journals of Gerontology Series B: Psychological Sciences and Social Sciences. 2010; 65B(3):331-9. [DOI:10.1093/geronb/gbq006]

[35] Freeman AT, Santini ZI, Tyrovolas S, Rummel-Kluge C, Haro JM, Koyanagi A. Negative perceptions of ageing predict the onset and persistence of depression and anxiety: Findings from a prospective analysis of the Irish Longitudinal Study on Ageing (TILDA). Journal of Affective Disorders. 2016; 199:1328. [DOI:10.1016/j.jad.2016.03.042] 